\title{
On Delay versus Congestion in Designing Rearrangeable Multihop Lightwave Networks"
}

\author{
Valter Boljunčić ${ }^{1}$, Darko Skorin-Kapov² and Jadranka Skorin-Kapov ${ }^{3}$ \\ ${ }^{1}$ Faculty of Economics and Tourism "Dr. Mijo Mirković", Pula, University of Rijeka, Croatia, \\ Fulbright Scholar, State University of New York at Stony Brook, USA \\ 2 School of Business, Adelphi University, Garden City, NY, USA \\ ${ }^{3}$ W. A. Harriman School for Management and Policy, State University of New York at Stony Brook, USA
}

\begin{abstract}
We investigate design issues of optical networks in light of two conflicting criteria: throughput maximization (or, equivalently, congestion minimization) versus delay minimization. We assume the network has an arbitrary topology, the flow can be split and sent via different routes, and it can be transferred via intermediate nodes. Tabu search heuristic is used to compare solutions with different weights assigned to each of the two criteria. The approach is tested on a benchmark data set, the 14-dimensional NSFNET T1 network with traffic from 1993. The results suggest that (1) some connectivity matrices are quite robust and desirable regarding both criteria simultaneously; (2) forcing minimization of total delay unconditionally can result with significantly inferior throughput. Some decisions strategies are outlined.
\end{abstract}

Keywords: heuristic solvability, tabu search, multihop, rearrangeable optical networks, minimal delay, maximal throughput

\section{Introduction}

Despite a surge in wireless communication, optical networks are still indispensable as a fast and reliable medium for transferring high volume sensitive data. A possibility for reconfiguration by re-tuning node transmitters and receivers to different wavelengths adds to usefulness and economic benefits of optical networks. The underlying assumption is that each network node is equipped with a small number $p$ of transmitters and receivers, and that a spectrum of wavelengths is accessible by and shared among all nodes using Wavelength Division Multiplexing (WDM). In order for traffic to go through a link $(i, j)$, a transmitter of node $i$ and a receiver of node $j$ have to be tuned to the same wavelength. Such a tuning establishes a logical link. Re-tuning results in new paths, making logical connectivity independent of physical architecture. This approach offers possibilities for optimizing logical connections in light of changes in incoming traffic. Depending of input traffic flow, the optical network can be optimized by taking into account different criteria, such as throughput, delay, or total flow. Relevant research considers arbitrary as well as regular network topologies (see SkorinKapov and Labourdette, 1996 and 1998). This work deals with arbitrary networks since they are more general and exist for every network size.

A discussion of different design objectives is presented in [Labourdette, 1998]. Previous work always considered one criterion at a time. The exception is the recent work by [Boljunčić, Skorin-Kapov and Skorin-Kapov, 2001] that investigates throughput maximization versus minimization of total network flow. The work presented in this paper deals with joint consider-

\footnotetext{
*The work was partially supported by the National Science Foundation grant ANI-9814014, by the project 036033- Architectural Elements for Regional Information Infrastructure, funded jointly by the Ministry of Science and Technology of the Republic of Croatia and the Istrian County, and by the project 067010- Models and Methods of Operational Research funded by the Ministry of Science and Technology of the Republic of Croatia.
} 
ation of network delay and congestion. Obviously, in the process of minimizing the maximal congestion the traffic will be re-routed, in turn using longer paths contributing to overall transmission delay. On the other hand, smaller congestion results in smaller queuing delay since the throughput is increased. Hence, it makes sense to investigate the behaviour of the network routing in light of joint consideration of minimal congestion and minimal delay. Regarding congestion, it makes sense to minimize maximal congestion because it increases network scalability and robustness regarding possible bottlenecks. The appropriate consideration of network delay should take into account managerial issues: is it more appropriate to consider minimizing maximal or total delay? (Minimization of average delay is accomplished by minimizing the total delay.) Minimizing total delay leads to overall better network performance. In addition, since the input flow from $i$ to $j$ can be split and sent via different routes using intermediate nodes, it can contribute to a total flow on any link.

This paper investigates different virtual designs and respective routings of optical networks obtained by joint consideration of minimizing con- gestion and total delay. The heuristic approach based on tabu search follows the strategy proposed by [Skorin-Kapov and Labourdette, 1995]. This requires decomposing the problem into two subproblems: the connectivity and the routing problems. The initial connectivity diagram (i.e. virtual topology) is obtained via a linear assignment problem that seeks a $0 / 1$ solution which maximizes the one hop path traffic. For a given connectivity matrix, a routing problem is a multicommodity flow problem with the objective function that is a weighted sum of both criteria. A local search based on branch exchange and tabu strategy is used to explore different virtual topologies. Computational results presented in this paper include a benchmark network data set of size 14 , with $p=2,3,4$ transceivers per node. A 14-node network NSFNET is displayed in Figure 1.

The paper is organized as follows. The formulation of our problem is presented in Section 2, an outline of the tabu search algorithm is given in Section 3, and the computational results are presented in Section 4. Future research is indicated in Section 5.

\section{NSFNET T1 Network 1991}

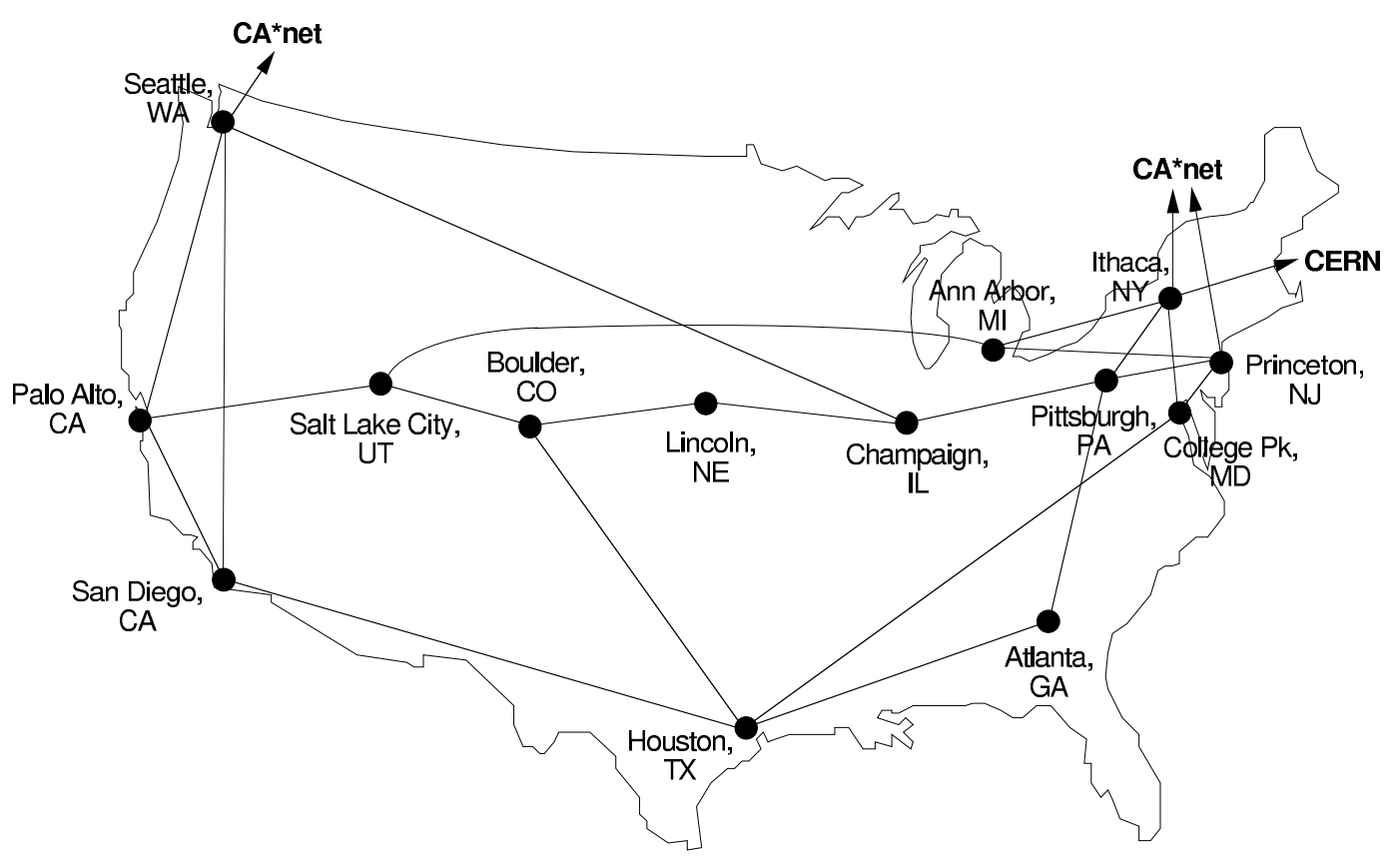




\section{Problem Formulation}

Our formulation is a modification of the minimal congestion problem entitled The Flow and Wavelength Assignment (FWA) problem, previously considered in a number of studies including [Labourdette and Acampora, 1991], [Yener and Boult, 1994], [Bienstock and Günlük, 1995], and [Skorin-Kapov and Labourdette, 1995].

The input $N \times N$ traffic matrix $T_{s t}$ presents the traffic flow from source $s$ to destination $t$, while $d_{s t}$ represents the distance (in miles) between nodes $s$ and $t$. For any node, the number of transmitters and receivers is set to $p$, and the capacity of every link equals $C$. (For simplicity, the capacities of all channels are assumed to be equal and large enough, so that the feasible solution exists.) The variables include $\{0,1\}$ variables $z_{i j}$ indicating whether or not a link $(i, j)$ is used in the network, and continuous variables $f_{k i j}$ indicating the amount of flow originating at source $k$, sent through the link $(i, j)$. Our objective is congestion minimization, as well as minimization of the total delay when sending the flow through the network.

The problem is formulated as follows:

$$
\min \omega_{F} F+\omega_{D} D
$$

s.t.

$$
\begin{aligned}
& \sum_{k, i, j} f_{k i j} d_{i j} \leq D \\
& \sum_{k} f_{k i j} \leq F \quad \text { for all } i, j, i \neq j \\
& \sum_{k} f_{k i j} \leq C z_{i j} \text { for all } i, j, i \neq j \\
& \sum_{i \neq j} f_{k i j}-\sum_{i \neq j} f_{k j i}=T_{k j} \text { for all } k, j, k \neq j \\
& \sum_{j \neq i} z_{i j}=p, \text { for all } i \\
& \sum_{j \neq i} z_{j i}=p, \text { for all } i \\
& 0 \leq f_{k i j}, \quad z_{i j} \in\{0,1\}, \text { for all } k, i, j, i \neq j
\end{aligned}
$$

The equation (1) models minimization of the total network delay using $\omega_{D}$ as the weight in the objective function. The equation (2) models minimization of maximal flow on any link (i.e. congestion) with weight $\left(\omega_{F}\right)$ in the objective function. The equation (3) enforces the capacity constraints on links, (4) are conservation of flow constraints, and $(5,6)$ are assignment type constraints assuring $p$ transmitters and receivers on any node.

In order to get a starting topology, we first solve a linear assignment problem maximizing the one hop path traffic (see [Skorin-Kapov and Labourdette, 1995]):

$$
\max \sum_{i j} T_{i j} z_{i j}
$$

s.t.

$$
\begin{gathered}
\sum_{j \neq i} z_{i j}=p, \text { for all } i \\
\sum_{j \neq i} z_{j i}=p, \text { for all } i \\
0 \leq z_{i j} \leq 1 \text { for all } i, j, i \neq j .
\end{gathered}
$$

Sometimes it might be desirable to start with a connectivity matrix known to be good from previous search. In that case we can proceed directly to the routing part of the problem. Namely, the values of the connectivity matrix, $\overline{z_{i j}}$, are used in the formulation of the routing multi-commodity flow problem:

$$
\min \omega_{F} F+\omega_{D} D
$$

s.t.

$$
\begin{gathered}
\sum_{k, i, j} f_{k i j} d_{i j} \leq D \\
\sum_{k} f_{k i j} \leq \overline{z_{i j}} F \quad \text { for all } i, j, i \neq j \\
\sum_{i \neq j} f_{k i j}-\sum_{i \neq j} f_{k j i}=T_{k j} \text { for all } k, j, k \neq j \\
0 \leq f_{k i j}, \text { for all } k, i, j, i \neq j
\end{gathered}
$$

Further improvements in solution quality are obtained via a tabu search heuristic strategy. In this paper we follow the tabu search strategy presented in [Skorin-Kapov and Labourdette, 1995]. 


\section{Tabu Search Heuristic Strategy}

Our solution is presented as (con, rout, $F, D)$, where con denotes a connectivity matrix, rout is the corresponding matrix of flow values, $F$ is the maximal flow value, and $D$ is the total network delay. In order to employ a tabu search heuristic strategy, we need to define a neighborhood of a current solution and ways to evaluate it. For our problem, a neighbor of the current solution (con, rout, $F, D$ ) is the feasible solution obtained by performing a branch-exchange (BE) operation, and by re-solving the routing problem. Hence, two neighboring connectivity topologies will differ in two branches and the corresponding routing which can be modified throughout the whole network. Due to the combinatorial nature of the connectivity part of the problem, evaluation of the complete neighborhood is computationally intractable. This is the reason behind the restriction to evaluate only a subset of branch exchanges. We elect to evaluate branch exchanges on $K$ least utilized links which will, nonetheless, preserve network connectivity. An iteration of the search is completed when the $K$-neighborhood of a current solution is evaluated, and the non-tabu neighboring solution with the smallest objective value is identified and performed. The tabu list is implemented as an $N \times N$ matrix whose $(i, j)$ entry denotes the iteration at which the link $(i, j)$ is no longer tabu. We start with the matrix of 0 's, i.e. all links can be exchanged at first iteration. If at iteration $I$ the old links $(i, j)$ and $(k, l)$ are replaced by the new links $(i, l)$ and $(k, j)$, then, in order to forbid reversal of this branch exchange for the tabu_size number of iterations, we update the matrix as follows: tabu_list $(i, j)=I+$ tabu_size; tabu_list $(k, l)=I+t a b u$ size. The tabu status of a link is inactive only if the branch exchange including this link leads to a solution better than the best found previously.

Tabu search was enhanced by a diversification strategy employed via a long term memory (LTM) function that constructs new initial solutions significantly different from previously visited solutions. The diversification was achieved by modifying the original "flow" entries, but at the same time this strategy had elements of intensification as well, since modifications were geared towards promising solutions (as encountered on the search trajectory). In addition, the tabu search strategy was further enhanced via repeated runs starting with good quality connectivity matrices from previous search.

\section{Computational Results}

The tabu search algorithm was coded in C, and the routing subproblems were solved using Cplex 7.0 callable library from [ILOG, 2000]. The computational experiments were performed on a PC. The benchmark data set used is the 14-dimensional matrix from [Mukherjee et al., 1996] with $p=2,3,4$ transceivers. This data consists of bytes/sec and is an actual measurement of the traffic on the T1 NSFNET backbone for a 15 -minute period (11:45pm to midnight) on January 12, 1993.

We start with the $100 \%$ weight given to congestion minimization and $0 \%$ weight given to minimization of total delay. The consecutive weights were modified in decrements (resp., increments) of $10 \%$ for congestion (resp., total delay). During the first tabu search run, the best connectivity matrix from the previous step was used as a starting solution in the next step. The best connectivity matrix for each pair of weights was saved. The interesting finding revealed that a small number of connectivity matrices repeatedly proved best over a large range of weight pairs. This motivated the second tabu search run, re-started with the connectivity matrix that appeared to be the best in majority of the cases. The results are presented in Table 1. Parameters of tabu search are as follows: $\mathrm{K}=8$, Tabu-size $=4$, Max-iterations $=30$, LTMrestart $=4$. Variable tabu-size with $\delta=[1,4]$ was also applied according to the strategy presented in [Skorin-Kapov and Labourdette, 1995].

Results from Table 1 reveal the following. As the weight for congestion minimization weakens from $\omega_{F}=1$ towards $\omega_{F}=0$, the congestion increases showing some significant jumps. At the same time, as the weight for total delay increases from $\omega_{D}=0$ to $\omega_{D}=1$, it is causing decrease in total delay. The decrease of delay includes less drastic jumps, and for $p=4$ it is distributed more uniformly. (The increase, 


\begin{tabular}{||cc|cc|cc|cc||}
\hline \hline \multicolumn{2}{|c|}{$\begin{array}{c}\text { weights } \\
\omega_{F}\end{array} \omega_{D}$} & $\begin{array}{c}\text { \% Congestion } \\
\text { increase }\end{array}$ & $\begin{array}{c}\text { \% Delay } \\
\text { decrease }\end{array}$ & $\begin{array}{c}\mathrm{p}=3 \\
\text { \% Congestion } \\
\text { increase }\end{array}$ & $\begin{array}{c}\text { \% Delay } \\
\text { decrease }\end{array}$ & $\begin{array}{c}\text { \% Congestion } \\
\text { increase }\end{array}$ & $\begin{array}{c}\text { \% Delay } \\
\text { decrease }\end{array}$ \\
\hline \hline 1.0 & 0.0 & 0.00 & 0.00 & 0.00 & 0.00 & 0.00 & 0.00 \\
0.9 & 0.1 & 2.14 & 19.02 & 2.70 & 8.19 & 0.00 & 0.00 \\
0.8 & 0.2 & 2.14 & 19.02 & 3.19 & 8.91 & 0.88 & 1.29 \\
0.7 & 0.3 & 12.57 & 26.62 & 10.25 & 16.82 & 1.74 & 2.04 \\
0.6 & 0.4 & 24.28 & 33.24 & 13.86 & 18.78 & 3.27 & 2.87 \\
0.5 & 0.5 & 25.86 & 33.72 & 18.69 & 20.51 & 7.97 & 4.48 \\
0.4 & 0.6 & 27.48 & 34.04 & 22.82 & 21.51 & 18.23 & 6.71 \\
0.3 & 0.7 & 28.31 & 34.16 & 42.59 & 24.29 & 21.51 & 7.27 \\
0.2 & 0.8 & 67.00 & 37.99 & 56.01 & 25.64 & 27.38 & 7.84 \\
0.1 & 0.9 & 85.74 & 38.80 & 64.86 & 26.02 & 47.02 & 8.90 \\
0.0 & 1.0 & 110.66 & 39.08 & 141.71 & 26.52 & 144.71 & 9.98 \\
\hline \hline
\end{tabular}

Table 1. Results for Infocom94 data set from [Mukherjee at al., 1996].

resp. decrease, is relative to the initial value for the pair $\left.\left(\omega_{F}, \omega_{D}\right)=(1,0).\right)$

The total percent increase in congestion is significantly bigger than the total percent decrease in total delay. The intuition is that the objective of minimizing the congestion allows for less flexibility in attaining it and, hence, forces solutions to change more significantly when its weight is changed. The network manager should decide how much congestion needs to be sacrificed in order to bring down the total network delay. Our analysis can identify points where more drastic changes take place, in turn helping the manager to make an economically justified decision.

\section{Conclusions and Directions for Further Research}

This paper presents our approach towards designing optical networks with desirable properties regarding congestion minimization as well as total delay minimization. Our future work will emphasize improvements in the basic tabu search strategy employed in order to identify efficient virtual topologies. Furthermore, we will investigate congestion versus delay for networks with regular underlying topologies. Relevant work was done by [Skorin-Kapov and Labourdette, 1996, 1998].

\section{References}

[1] D. BIEnstock AND O. GÜNLÜK, (1995), "Computational experience with a difficult mixed-integer multicommodity flow problem", Mathematical Programming 68, 213-237.

[2] V. BOLJUnČIĆ, D. SKORIN-KAPOV AND J. SKORINKAPOV, (2001), "A Design of Rearrangeable Lightwave Networks: A Multicriteria Approach", Working Paper, State University of New York at Stony Brook.

[3] “CPLEX 7.0” (2000), ILOG, Inc.

[4] J.-F. LABOURDETTE, (1998), “Traffic optimization and dynamic reconfiguration management of multiwavelength lightwave networks", Computer Networks and ISDN Systems 30, 981-998, 1998.

[5] J-F. LABourdetTE AND A. ACAMPORA, (1991), "Logically Rearrangeable Multihop Lightwave Networks", IEEE Transactions on Communication 39, 1223-1230.

[6] B. MukHerJee, S. RAmmamurthy, D. BANERJEe, AND A. MuKHERJE, (1996), "Some Principles for designing a wide-area WDM optical network", IEEE/ACM Transactions on Networking, 4(5), 684696.

[7] J. SKORIN-KaPOV AND J.-F. LABOURDETTE, (1995), "On Minimum Congestion Routing in Rearrangeable Multihop Lightwave Networks", Journal of Heuristics, 1(1), 129-145. 
[8] J. SKORIN-KAPOV AND J.-F. LABOURDETTE, (1996), "On Throughput Optimization in Optical Networks with Regular and Arbitrary Topologies", Journal of Computing and Information Technology, 5(4), 219-230.

[9] J. SKORIN-KAPOV AND J.-F. LABOURDETTE, (1998), "Rearrangeable Multihop Lightwave Networks: Congestion Minimization on Regular Topologies", Telecommunication systems $9,113-132$.

[10] B. YenER AND E. Boult (1994), “A study of upper and lower bounds for minimum congestion routing in lightwave networks", Proceedings of Infocom'94, Toronto, Canada.

Received: June, 2001 Accepted: September, 2001

Contact address:

Valter Boljunčić Faculty of Economics and Tourism "Dr. Mijo Mirkovíc"

University of Rijeka

Preradovićeva $1 / 1$

52100 Pula, Croatia e-mail: vbolj@efpu.hr

Fulbright Scholar, State University of New York at Stony Brook, USA e-mail: vboljuncic@notes.c|c.sunysb.edu

Darko Skorin-Kapov

School of Business, Adelphi University Garden City, NY, 11530, USA e-mail: skorin@adelphi.edu

Jadranka Skorin-Kapov

W.A. Harriman School for Management and Policy State University of New York at Stony Brook Stony Brook, NY 11794-3775, USA

Phone: +1-631-632-7426

Fax: +1-631-632-9412

e-mail: jskorin@notes.c|c.sunysb.edu

Web: http://www.sunysb.edu/harriman/jadranka.htm
VALTER BOLJUNČIĆ received his B.Sc. (1983), M.Sc. (1994) and Ph.D. (1999) degrees in mathematics from the University of Zagreb. He is currently Assistant Professor at the Faculty of Economics and Tourism in Pula, University of Rijeka. His research interests include efficiency and sensitivity analyses. He spent the academic year 2000./01. as a Fulbright scholar at SUNY at Stony Brook doing research in efficiency and layout of optical telecommunication networks.

DARKO SKORIN-KAPOV is an Associate Professor of Information Technology in the School of Business, Adelphi University, Garden City, New York. He got his B.Sc. and M.Sc. degrees in Mathematics and Information Science from the University of Zagreb, Croatia. He received his $\mathrm{Ph} . \mathrm{D}$. in Management Science from the University of British Columbia, Vancouver, B.C., Canada. His research is predominantly in the area of network optimization and cost allocation. He has extensive academic, as well as, industrial experience in those areas. His publications include papers in Annals of OR, Discrete Applied Mathematics, European Journal of OR, Location Science, Management Science, Networks, OR Letters, SIAM Journal of Discrete Mathematics and Telecommunication Systems.

JADRANKA SKORIN-KAPOV received her B.Sc. (1977) and M.Sc. (1983) degrees in Applied Mathematics from the University of Zagreb, and her Ph.D. (1987) in Operations Research from the University of British Columbia, Canada. She is currently a Professor in the W.A. Harriman School for Management and Policy, State University of New York at Stony Brook. Her research interests include combinatorial optimization, nonlinear programming, and development of solution procedures for difficult optimization problems arising in telecommunications, manufacturing, and facility layout and location. She has received five National Science Foundation grants and has published extensively, in cluding articles in Mathematical Programming, Operations research Letters, ORSA Journal on Computing, Computers and Operations Research, Discrete Applied Mathematics, Telecommunication Systems, Journal of Computing and Information Technology, European Journal of Operational research, and Annals of Operations Research. 